\title{
Birth of the programmable optical chip
}

\author{
Advances in silicon photonics, compound III-V semiconductor technology and hybrid integration now \\ mean that powerful, programmable optical integrated circuits could be within sight.
}

The recent acquisition of Altera, the pioneer of programmable logic chips, for US $\$ 16.7$ billion by the wellknown chip maker Intel provides clear recognition of the perceived importance of field-programmable gate array (FPGA) technology. In essence, an FPGA chip is a universal signal processing chip that can be programmed or configured after fabrication to perform a specific task - be it speech recognition, computer vision, cryptography, or something else.

Originally commercialized in the mid-1980s, by two US Silicon Valley firms Altera and Xilinx (who today between them hold an $\sim 80 \%$ share of the market), the FPGA chip has grown from humble origins and niche applications to ubiquity. The technology is found inside everything from digital cameras and mobile phones through to sophisticated medical imaging devices, telecommunications equipment and robotics. In the heart of an FPGA is a large array of logic blocks that are wired up by reconfigurable interconnects, allowing the chip to be reconfigured or programmed via specialized software. The use of a standard common hardware platform makes FPGAs far more flexible and cost effective compared with application specific integrated circuits (ASICs) - complex chips that are custom designed for a specific task.

What's potentially exciting is that there are now signs that the optical equivalent of an FPGA is on the horizon. Improvements in both silicon photonics and III-V compound semiconductor technology, such as InP and GaAs, mean that optical researchers are starting to build designs of programmable optical signal processors on a chip by cascading arrays of coupled waveguide structures that feature phase shifters to control the flow of light through the array and thus support reconfigurability. The theory of how such arrays behave has been analysed in depth by David Miller from Stanford University in the US who has published several papers on the topic.

Research teams around the globe specializing in microwave photonics, including Jianping Yao's group at the University of Ottawa, Canada, José Capmany's at Valencia, Spain and Arthur Lowery's group at Monash University in Australia, and others are building

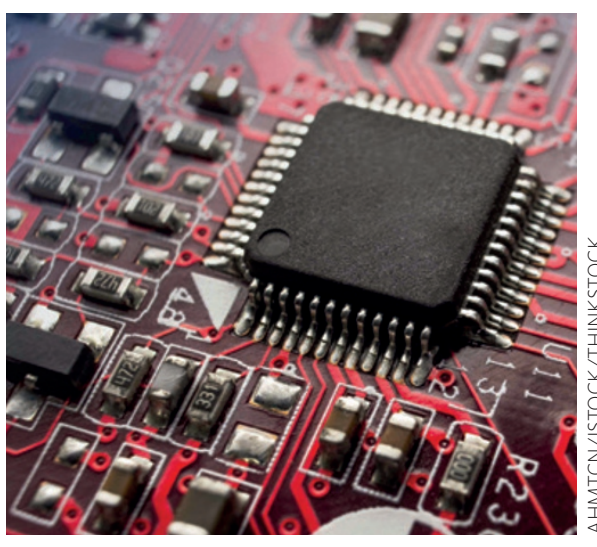

is leading to a situation where there are almost as many technologies as there are applications. This fragmentation hinders cost-effective, mass-volume manufacture of a photonic solution, a situation that an optical programmable chip would help remedy. "It would be a considerable landmark because it would open the possibility of using the same hardware configuration, and thus reduce fabrication costs, to implement different functionalities by suitable programming," commented Capmany.

According to Capmany, the approach could also prove useful in other areas too, such as realizing a multifunctional lab on a chip in biophotonics, reconfigurable logic gates in quantum information systems and software-defined reconfigurable switching and routing fabrics in optical communication networks.

Capmany says that the key decision to be made is the best choice of a material platform for realizing such a chip. "We do have several technologies available but none seem to cover the full range of requirements. Indium phosphide is the only technology capable of providing active elements like lasers and amplifiers. It is certainly the most complete in terms of available components but is quite lossy," he commented. "Silicon brings compatibility with CMOS fabrication processes and low-loss passive components. Unfortunately it is not suitable for implementing optical sources and amplifiers. Finally, silicon nitride provides extremely low losses, which is good for passive components but it is not suitable for implementing active devices, detectors and modulators. It is still not clear if the best solution will be a monolithic or hybrid approach."

Regardless of which platform wins the day, Miller is certainly optimistic about the future potential. "We are really at the edge of a change in the way we think about optics, from thinking in terms of single devices and very simple circuits to systems in which we let a little additional complexity and smartness make the whole system easier to fabricate and operate and much more flexible," he comments. "We went through that transition in electronics some time ago, but we can now start to see how to make that transition in optics as well." 